im Ostsudan operierenden Beja Congress von Bedeutung, der von ihr militärisch unterstützt wird. Durch diese Unterstützung ist die SPLA in Kämpfe mit den SAF im Grenzgebiet zu Eritrea, insbesondere in der Gegend um Kassala, involviert. Dort kann sie um das Jahr 2000 einige militärische Erfolge erzielen.

Mit dem Erteilen dieser für den Norden schmerzhaften Nadelstiche geht ein interner Einigungsprozess einher. Riek Machar verlässt die SSDF, die sich in weiterer Folge unter dem Kommando von Paulino Matip auf den Schutz der Ölfelder in Unity State konzentriert (ebd.: 696). Er gründet kurzfristig die Sudan People's Defence Forces (SPDF), die ihm aber letztlich nur als Instrument zur Verhandlung seiner Reintegration in die SPLM/A dienen. Die Reintegration verläuft in der Tat zügig und wird im Januar 2002 abgeschlossen. Riek Machar ist nun wieder nominell die Nummer Drei jener Organisation, die er elf Jahre zuvor im Disput verlassen hat. Auch Lam Akol schließt sich mit seiner SPLM-Unity im Jahr 2004 wieder der SPLM/A an.

Von einer durchschlagenden militärischen Dominanz kann dennoch auf keiner der beiden Seiten gesprochen werden. Die SAF und die mit ihnen verbündeten Milizen kontrollieren nach wie vor die drei wesentlichsten Städte Juba, Wau und Malakal, in denen sich ihre militärische Schlagkraft bündelt. Die SSDF-Milizen in Unity State sind ebenfalls ein relevanter Faktor auf nordsudanesischer Seite. Im Sinne eines Vergleichs der militärischen Kräfte waren damit Anfang der 2000er-Jahre jene Bedingungen erfüllt, die I. William Zartman (2000) als ein »mutually hurting stalemate« bezeichnet hat, das im Sinne von Zartmans Begriff der »ripeness« - reif für eine Friedenslösung wäre. Dieses beide Seiten schmerzende Kräftegleichgewicht hätte theoretisch allerdings schon Mitte der 1980er-Jahre festgestellt werden können. Dies unterstreicht, dass es nicht allein für das wechselseitige Interesse an Friedensverhandlungen und deren letztendlichen Erfolg verantwortlich zu machen ist.

\title{
Ein umfassendes Friedensabkommen
}

Es sind zwei weitere Faktoren, einer ökonomisch, einer geopolitisch, die insbesondere die sudanesische Seite zum Eintritt in ernsthafte Verhandlungen motivieren. Der ökonomische Faktor, der schon zuvor den militärstrategischen Zugang Khartums bestimmt, ist die Ölförderung. In der zweiten Hälfte der 1990er-Jahre nahm die Ölexploration, vor allem in der Region um Bentiu, massiv zu. Im Jahr 1999 wird endlich die lange erwartete Pipeline von den Öl- 
feldern nach Port Sudan am Roten Meer eröffnet, die sofort die Realisierung substanzieller Ölrenten ermöglicht. Geradezu selbstverständlich werden diese Einnahmen in die Erneuerung und Verbesserung der Bewaffnung der SAF investiert. Zugleich führt die Karbonisierung des sudanesischen politischen Marktplatzes zu neuen politisch-ökonomischen Dynamiken.

"Sudan enjoyed an economic boom in the 2000s. The national budget expanded from $\$ 950$ million in 1999 to over $\$ 11$ billion in 2006. However, this petroleum-and-peace dividend was dispensed mostly on expanding the public sector and security services payroll. This was the NCP's means of consolidating its fractious constituency.« (de Waal, 2014: 353-354)

Diese doppelte positive Perspektive, die Ruhigstellung der Opposition im Inneren in Kombination mit der Option auf eine Verstetigung, wenn nicht sogar Verstärkung des ökonomischen Booms schaffen erst die internen Voraussetzungen für das Bashir-Regime, sich auf einen Verhandlungsprozess mit der SPLM/A einzulassen.

Eine weitere, nicht unwesentliche Motivation liegt in der unmittelbaren Bedrohung dieser eigentlich komfortablen Position durch die USA. Aus deren Sicht stellt die durch al-Turabi in der Regierung verkörperte NIF eine Bedrohung der internationalen Sicherheit dar. So wohnt etwa al-Qaeda-Gründer Osama bin Laden auf Einladung von al-Turabi von 1991 bis 1996 in Khartum. Auch weitere al-Qaeda-Größen halten sich wiederholt im Land auf. Sogar paramilitärische Trainingszentren für islamistische Kämpfer:innen werden auf sudanesischem Territorium eingerichtet. Die USA reagieren martialisch. Am 20. August 1998 schlägt eine US-amerikanische Cruise-Missile im Rahmen der Operation »Infinite Reach « in der Al-Shifa-Pharmafabrik in Khartum ein und zerstört diese. Dieser Angriff kommt für das Bashir-Regime einem ernst zu nehmendem Warnschuss gleich. In der Folge der Anschläge von 9/11 gewinnt der Druck der USA nochmals an Vehemenz. Dies lässt Bashir ernsthaft um seine Herrschaft fürchten und trägt zu seinem Zerwürfnis mit al-Turabi bei. Die NIF wird aus der Regierung ausgeschlossen. Angesichts dieser sich intern drastisch zuspitzenden Situation - der Darfur-Konflikt steht kurz vor einer beispiellosen Eskalation - bieten Friedensverhandlungen mit der SPLM/A die Möglichkeit einer willkommenen politischen Entlastungsoffensive.

Eingeleitet und betrieben wird der Friedensprozess von der im Jahr 1986 gegründeten Intergovernmental Authority on Development (IGAD). IGAD ist zu Beginn der Verhandlungsrunden zwischen der SPLM/A und dem Regime in Khartum noch eine kleine und eher obskur erscheinende Internationale Or- 
ganisation, bestehend aus Äthiopien, Dschibuti, Kenia, Somalia, dem Sudan und Uganda, sowie dem 1993 beitretenden, gerade unabhängig gewordenen Eritrea. Die IGAD beginnt ihr Engagement mit der Bündelung vorangegangener Prozesse. Die noch auf eine nigerianische OAU-Initiative zurückgehenden Verhandlungen in Abuja hatten im Mai 1994 eine Prinzipien-Deklaration hervorgebracht, die IGAD Declaration of Principles (DoP $)^{7}$, die so nach einigen Jahren der Bedeutungslosigkeit im Jahr 1997, im Zuge der Verhandlungen um das Khartoum Agreement, wieder relevant wird. IGAD betreibt die Einbindung der SPLM/A-Mehrheitsfraktion in die Gespräche mit Khartum.

Beim IGAD-Gipfel im Juli 1997 akzeptiert Bashir auf Druck des kenianischen Präsidenten Daniel Arap Moi die DoP als Basis zukünftiger Verhandlungen mit der SPLM/A (Murithi, 2009). Die Prinzipien ähneln dem Khartum-Prozess mit den abgespaltenen SPLM/A-Fraktionen: Waffenstillstand, Bekenntnis $\mathrm{zu}$ einer Verhandlungslösung, das ambivalente wechselseitige Zugeständnis von sowohl staatlicher Einheit und Selbstbestimmung mit einem Unabhängigkeits-Referendum als Ankerpunkt, und eine Interimsperiode als Vorbereitung von dessen Abhaltung. Dennoch ist eine Kompromissfindung gegenüber der SPLM/A für die sudanesische Seite um einiges schmerzhafter als gegenüber mit den mit ihr selbst verbündeten SPLM/A-Abspaltungen. Es gibt keine symbolischen Zugeständnisse wie die Akzeptanz einer Scharia-Gesetzgebung im ganzen Land. Auch ist offensichtlich, dass eine Vereinbarung mit der SPLM/A-Hauptströmung $\mathrm{zu}$ viel substanzielleren praktischen Konsequenzen führen wird. Ohne die oben geschilderten, für die Verhandlungen immer günstiger werdenden Rahmenbedingungen ist dieser Schritt kaum zu erklären.

Wiederum ist es von Daniel Arap Moi ausgeübter Druck auf beide Seiten, der die Aufnahme der ersten strukturierten Verhandlungen unter beidseitig akzeptierter Mediation der IGAD in Machakos nahe Nairobi ermöglicht. Als Chefverhandler fungiert der ehemalige kenianische Generalstabschef Lazaro Sumbeiywo. Sumbeiywo favorisiert einen pragmatischen, auf die Anliegen der Parteien fokussierten Stil und hält damit die internationale Einflussnahme, mit all den damit einhergehenden Vor- und Nachteilen, relativ gering. Ein Durchbruch gelingt. Am 20. Juli 2002 unterzeichnen Ghazi Salahuddin Atabani, Bashirs Berater für Friedensfragen, für die sudanesische Regierung und Salva Kiir für die SPLM/A das Machakos Protocol ${ }^{8}$. Im Wesentlichen bestä-

7 https://www.peaceagreements.org/viewmasterdocument/918.

8 https://www.peaceagreements.org/viewmasterdocument/440. 
tigt Machakos die Festlegungen der DoP, akzeptiert jedoch unter dem Banner von »freedom of belief, worship and conscience« implizit eine Anerkennung der Scharia. Eine sechsmonatige Prä-Interim-Periode wird vereinbart, gefolgt von einer sechsjährigen Interimsperiode, an deren Ende ein international überwachtes Referendum über die südsudanesische Unabhängigkeit stehen soll.

Unmittelbar nach der Unterzeichnung des Vertrages in Machakos verschärfen sich die Spannungen zwischen Garang und SPLM/A-Verhandlungsleiter Kiir, nicht zuletzt aufgrund Garangs eigener strategischer Ambivalenz. Er stößt sich an der starken Betonung der Selbstbestimmung in Machakos, verhandelt vom deklarierten Unabhängigkeitsbefürworter Kiir. »According to one person close to him at the time, >Garang went berserk«." (Young, 2012:94) Schließlich ersetzt Garang Kiir für die Folgeverhandlungen in Naivasha durch eine Reihe an erfahrenen Diplomaten: Nhial Deng Nhial, der in weiterer Folge als Chefverhandler agiert, Deng Alor Kuol und Pagan Amum Okiech. Nhial Deng, der Sohn des legendären Anya-nya-Mitbegründers William Deng Nhial, war bereits an der Aushandlung der DoP beteiligt und gilt als einer der besten Verhandler und Strategen der Bewegung. Pagan Amum agierte zuvor als zentraler Verbindungsmann zur NDA und Leiter der SPLA-Operationen im Nordsudan (Abol Kuok, 2015: 687, 693-694). Deng Alor wiederum ist ein deklarierter Politiker, der aufgrund seiner guten internationalen Verbindungen und, nicht zuletzt, seiner Herkunft aus dem von beiden Seiten beanspruchten Abyei zugezogen wird.

Die internen Spannungen, die in Berichten von einer angeblich bevorstehenden Entmachtung und Verhaftung Kiirs gipfeln, werden Ende November 2004 in einem SPLM-Meeting in Rumbek aufgearbeitet ${ }^{9}$. In seiner Ansprache während des Treffens bestreitet Garang eine Entmachtung Kiirs und verneint jede politische Spannung. Gleichzeitig verweist er aber auf den problematischen Charakter der expliziten Erwähnung von Selbstbestimmung im Machakos Protocol, mit dem Argument, dass dies für die sudanesische Seite schwer verdaulich wäre. Streit oder nicht: Faktum bleibt, dass Kiir in den folgenden Detailverhandlungen nicht mehr beigezogen wird.

Die Unterzeichnung des Machakos Protocol zeitigt sofortige militärische und politische Auswirkungen. Zunächst kommt es zu Kämpfen um Torit, das von der SPLM/A im September eingenommen und von SAF-Truppen im Oktober zurückerobert wird. Der Sinn dieser Gefechte, die den Friedensprozess 
kurzfristig an den Rand des Scheiterns bringen, ist umstritten. John Young (2012: 101) vermutet dahinter den Versuch der SPLM/A, ihre Verhandlungsposition zu verbessern. Die Attacke auf Torit ist in der Tat für das Regime in Khartum schmerzhaft. Zahlreiche hochrangige SAF-Militärs finden in den Kämpfen den Tod, darunter persönliche Freunde von al-Bashir. Am 15. Oktober 2002 wird allerdings der Fortgang der Verhandlungen beschlossen und endgültig ein Waffenstillstand unterzeichnet ${ }^{10}$, der bis zum Ende der Verhandlungen halten sollte.

Auch die internationale Ebene gerät in Bewegung. Bereits im Oktober 2000 beginnt im Rahmen eines Treffens des IGAD Partner Forums in London ein engerer Austausch zwischen den USA, angeführt vom Afrika-Spezialisten John Prendergast vom US State Department, Großbritannien und Norwegen, das in der Gründung einer informellen "Troika« mündet. Mit dem Machtwechsel in den USA zur republikanischen Präsidentschaft von George W. Bush im Januar 2001 nimmt deren Sudan-Politik eine Wende zu einer gesteigerten Einflussnahme, was zunächst durch die Verbindung Bushs zu evangelikalen Gruppen und bald durch die Anschläge von 9/11 bedingt ist (Johnson, 2011: 25). Die USA sind gewillt, die Troika aktiv zur Forcierung der Verhandlungen einzusetzen. Ein Sonderbeauftragter, John Danforth, ein methodistischer Pastor, wird eingesetzt (Rolandsen und Daly, 2016: 134). Unter seiner Initiative entwickelt sich das informelle Bündnis zu einer tragenden Säule der internationalen Unterstützung des Friedensprozesses.

Die nächsten Verhandlungsrunden finden in Naivasha, einer ebenfalls in Kenia nahe Nairobi gelegenen touristischen Metropole, statt. Es werden insgesamt sechs weitere Protokolle ausverhandelt und unterschrieben: Ende September 2003 machen die Security Arrangements den Anfang. Sie bauen auf vorangegangenen Verhandlungsresultaten auf und sehen in erster Linie den Rückzug der SAF hinter die Nord-Süd-Grenze zum Zeitpunkt der sudanesischen Unabhängigkeit sowie die Einrichtung sogenannter Joint Integrated Units (JIU) vor. Diese JIUs sollen insgesamt 39.000 Soldat:innen umfassen, die meisten davon (24.000) stationiert im Süden, jeweils 6.000 in den zwei umstrittenen Grenzregionen Blue Nile und Nuba Mountains, und 3.000 in der Hauptstadt Khartum. Die Leitung obliegt einem gemeinsam eingerichteten Joint Defence Board (JDB).

10 Memorandum of Understanding on Cessation of Hostilities Between the Covernment of the Sudan and the Sudan People's Liberation Movement/Army; https://www.peacea greements.org/viewmasterdocument/453. 
Bereits im Januar 2004, und damit überraschend schnell, kommt es zu einer Einigung zur Ressourcenteilung (»Wealth Sharing«). Das herausfordernde Thema ist die Aufteilung der Einkommen aus der Ölförderung. Es wird eine 50:50-Teilung vereinbart, wobei $2 \%$ der Gesamteinnahmen den lokalen Verwaltungen in den ölfördernden Regionen zukommen müssen. Eine paritätisch besetzte National Petroleum Commission (NPC) wird eingerichtet, die sich um auftretende Detailprobleme kümmern soll. Und diese wird es schnell geben. Was das CPA nicht regelt, sind die Gebühren für den Öltransport in der Pipeline nach Port Sudan, eine Frage, die sich in weiterer Folge zu einer ernsten Krise auswachsen wird.

Im Mai erfolgt die Unterzeichnung der Protokolle zur politischen Machtteilung und zum Status der umstrittenen Gebiete, Abyei, sowie Southern Kordofan und Blue Nile, den sogenannten »Two Areas«. Für Abyei wird ein Referendum zur Bestimmung der Zugehörigkeit der Region festgesetzt, abzuhalten am selben Tag wie das Unabhängigkeitsreferendum am Ende der Interimsperiode. Die Bestimmungen für die Two Areas bleiben hingegen weitgehend unbestimmt. Verwiesen wird auf "popular consultations « und die daran anschließende Einrichtung einer unabhängigen Kommission, was mehr oder minder der beidseitigen Akzeptanz eines Verbleibs dieser Regionen beim Norden gleichkommt.

Das Power-Sharing Protocol legt nicht nur einen Schlüssel für die Machtteilung in Exekutive und Legislative vor den am Ende des vierten Jahres der Interimsperiode angesetzten Wahlen fest. Es begründet eine südsudanesische Regionalregierung, das Government of South Sudan (GoSS). Der Präsident des GoSS besetzt in dieser Konfiguration einen »Doppelhut « mit der Position als Erster Vizepräsident des Sudan - diese Funktionen werden nach der Unterzeichnung des CPA von John Garang eingenommen, Salva Kiir wird zum Vizepräsidenten des GoSS ernannt. In Exekutive und Legislative wird eine prozentuale Aufteilung der Positionen festgelegt: $52 \%$ für die NCP, $28 \%$ für die SPLM, 14 \% für Oppositionsparteien aus dem Norden, und $6 \%$ für andere politische Kräfte aus dem Süden. Zu den zwei wichtigsten Ministerposten für die SPLM entwickeln sich das Außenressort und das Ministerium für Cabinet Affairs, die während der Interimsphase in wechselnden Rollen von Deng Alor und Lam Akol eingenommen werden.

Der Verhandlungsprozess verschärft die Auseinandersetzungen innerhalb des sudanesischen Regimes, die letztendlich in seiner Spaltung münden. Bashir entmachtet die islamistischen Kräfte um Turabi im März 2004 und lässt Turabi verhaften. Auf die Friedensverhandlungen hat dies, im Zusam- 
menhang mit den anderen, oben erwähnten Faktoren wie der zeitgleichen Zuspitzung der Krise in Darfur, positive Auswirkungen. Am 9. Januar 2005 unterschreiben John Garang und der sudanesische Vizepräsident Ali Osman Mohammed Taha nochmals alle Protokolle in zusammengezogener Form als Comprehensive Peace Agreement (CPA).

Generell regelt das Abkommen in pragmatischer Weise die zwei schlagkräftigsten Elemente des sudanesischen Marktplatzes, »money and guns « (de Waal, 2021: 334). In Bezug auf seine weitergehende politische Funktionalität reproduziert es die strategische Ambivalenz, die die politische Führung der SPLM/A schon seit Langem kennzeichnet. »[T]he text emerged from multiple different incompatible processes and the two principals were, even at the time of signing in January 2005, evasive about their ultimate goals. (de Waal, 2021: 321) Die zur narrativen Verankerung dieser Ambivalenz gewählte Phrase ist »making unity attractive«, die sich an zwei Stellen der Präambel findet und sich schnell zum geflügelten Wort entwickelt. Die exakte Formulierung liest sich wie ein politisches Zugeständnis des Regimes in Khartum, das zugleich der zweideutigen politischen Ausrichtung der SPLM/A entgegenkommt: der Vertrag »recognizes the right of the people of Southern Sudan to self-determination and seeks to make unity attractive during the Interim Period «. Gerade die exklusive Fokussierung auf die zwei unterzeichnenden Parteien widerspricht allerdings dem Gedanken, diese Attraktivität durch eine demokratische Transformation zu erreichen (Aalen, 2013; Young, 2012: 113-114).

Das CPA ist ein viel gescholtener Vertrag. Oft wird er despektierlich als ein überlanges Waffenstillstandsabkommen bezeichnet, oder als ein Versuch des Reinwaschens des sudanesischen Regimes von seinen Verbrechen in Darfur - und von den Jahrzehnten der verbrecherischen Kriegsführung im Süden. Dennoch sollte nicht vergessen werden, dass zum Zeitpunkt der Verhandlungen alle historischen Erfahrungen auf eine Weiterführung des Krieges hindeuten. $\mathrm{Zu}$ Recht bezeichnet Alex de Waal in seiner analytischen Nachbetrachtung den Vertrag daher als ein »achievement against the odds « (de Waal, 2021: 342). Selbst eingedenk der geopolitisch günstigen Voraussetzungen und des starken internationalen Drucks auf Khartum ist der politische Schritt von Omar al-Bashir, dem Süden den zu diesem Zeitpunkt zumindest potenziellen Weg zur Eigenstaatlichkeit freizugeben, von historischer Dimension. 